\title{
Chinese Journal of Cancer reviewer acknowledgement 2015
}

Rui-Hua Xu ${ }^{1}$ and Sarah M. Theissen ${ }^{2^{*}}$

\section{Contributing reviewers}

The Editors of Chinese Journal of Cancer would like to thank all our reviewers who have contributed to the journal in 2015.

Without the participation of skilful reviewers no academic journal could succeed and we are grateful to the committed individuals who have given their time and expertise to the peer review of manuscripts for Chinese Journal of Cancer. We look forward to your continued support in 2016.

Jin-Xin Bei

China

Qing-Qing Cai

China

\section{Zheng Cao}

USA

Sumei Cao

China

Xinping Cao

China

Ming Chen

China

Zhongping Chen

China

Hankui Chen

USA

Jun Chen

China

Hui Chen

China

Nianyong Chen

China

\author{
Hongyan Chen \\ China
}

Tao Chen

China

Ming-Yuan Chen

China

Qiong Chen

China

GuoQiang Chen
China

Jindong Chen

USA

Zhe-Sheng Chen

USA

Gong Chen

China

Xiaodong Cheng

China

Shi-Yuan Cheng

USA

Zhibin Cheng

China

\author{
Niall Corcoran \\ Canada
}

Yiwei Cui

China

Bingbing Dai

USA

Yun Dai

USA

Xin Du

China

Chaojun Duan

China

Yun Fan

China

Wei Fan

China

Qipeng Fan

USA

Liwu Fu

China

Xianshu Gao

China

*Correspondence: sarah.theissen@biomedcentral.com

2 BioMed Central, 236 Gray's Inn Road, London WC1X 8HB, UK

Full list of author information is available at the end of the article 
Song Gao

China

Guoquan Gao

China

Minghua Ge

China

Cuizhi Geng

China

Dongmin Gu

USA

Rongping Guo

China

Zhigang Guo

USA

Jun Guo

China

Mingzhou Guo

China

Hui Han

China

Xiaohui He

China

Biao He

USA

Jiang He

USA

Qiang He

China

Chaosu Hu

China

Zheyu Hu

USA

Xiaojun Huang

China

Zhouguang Hui

China

Hongbin Ji

China

Wei-Hua Jia

China

Lintao Jia

China
Tiebang Kang

China

Sanath Kumar

USA

Xiao Li Lan

China

Xiang-Ming Lao

China

Nan Li

USA

Jiancheng Li

China

Zhi-Ming Li

China

Yuhong Li

China

Xin-Jian Li

USA

Guancheng Li

China

Jinfeng Li

China

Bing Li

China

Jingao Li

China

Jinjun Li

China

Jin Li

China

Li-Li Li

Hong Kong

Qin Li

China

Chao Li

China

Hua Li

China

Jun Li

China

Yanfang Li

China
Shengping Li

China

Shaojun Lin

China

Qing Liu

China

Yueping Liu

China

Yongyu Liu

USA

Ran-Yi Liu

China

Yong-Feng Liu

China

Li Liu

China

Guoyan Liu

China

Hao Long

China

Herbert Loong

China

You Lu

China

Shun Lu

China

Junhang Luo

China

Yue Lv

China

Jie Ma

China

Jun Ma

China

Haiqiang Mai

China

Jaap Middeldorp

The Netherlands

Koji Mita

Japan

Yong-Gao Mou

China 
Jianyun Nie

China

Na Niu

USA

Jianji Pan

China

Han Peng

USA

Ji Peng

China

Francesco Pezzella

UK

Chao-Nan Qian

China

Haide Qin

USA

Shuangjian Qiu

China

Derek Raghavan

USA

Zefang Ren

China

Jun Ren

China

Maryam Sedghi

Iran

Jian-Yong Shao

China

Lin Shen

China

Kunwei Shen

China

Yanxia Shi

China

Zhi Shi

China

Yuqin Song

China

Ming Song

China

Tianqiang Song

China
Yan Sun

China

Min-Han Tan

Singapore

Yongguang Tao

China

Ling Tian

China

Dean Tian

China

Jinghai Wan

China

Yan Wang

USA

Lian-Tang Wang

China

Zhiqiang Wang

China

Jin Wang

China

Jianbing Wang

China

Shixuan Wang

China

Yongbo Wang

China

Yongming Wang

China

Zehua Wang

China

Yu Wang

China

Guiying Wang

China

Zhimin Wang

China

Xiang Wang

China

Joseph Wee

Singapore

Wei Wei

USA
Xiaohua Wu

China

Anhua Wu

China

Jiangxue Wu

China

Lingying Wu

China

Peihong Wu

China

Liangping Xia

China

Conghua Xie

China

Wanghong $\mathrm{Xu}$

China

Li Xu

China

Bing Xu

China

Xundi Xu

China

Li Yan

USA

Jian Yan

USA

Jilong Yang

China

Kunyu Yang

China

AnKui Yang

China

Ziming Yu

USA

Sheng-Ji Yu

China

Eugene Yu

Canada

Ping Yuan

China

Yuan Yuan

China 
Yan Yuan

USA

Musheng Zeng

China

Jian Zhang

China

L. Harris Zhang

USA

Li Zhang

China

Guo-Jun Zhang

China

Lanjun Zhang

China

Quan Zhang

China

Li Zhang

China

Jianing Zhang

China
Xiaoshi Zhang

China

Wen-Hao Zhang

China

Wei Zhang

USA

Ni Zhao

USA

Xianda Zhao

USA

Dinghai Zheng

USA

Dinghai Zheng

USA

Zhendong Zhong

USA

Chong Zhong

China

Zhiwei Zhou

China

Author details

${ }^{1}$ Department of Medical Oncology, Sun Yat-sen University Cancer Center, 651

Dongfeng Road East, Guangzhou 510060, Guangdong, China. ${ }^{2}$ BioMed Central,

236 Gray's Inn Road, London WC1X 8HB, UK.

Published online: 18 March 2016

Submit your next manuscript to BioMed Central and we will help you at every step:

- We accept pre-submission inquiries

- Our selector tool helps you to find the most relevant journal

- We provide round the clock customer support

- Convenient online submission

- Thorough peer review

- Inclusion in PubMed and all major indexing services

- Maximum visibility for your research

Submit your manuscript at www.biomedcentral.com/submit 